\title{
Effects of Bucumolol, a $\beta$-Adrenergic Blocking Agent, and Its d-Isomer on Myocardial Infarction Produced by Coronary Artery Ligation in Rats
}

\author{
Hiroyuki Korke, PhD., Seiji Kumakura, PhD., \\ Hiroshi Nishino, Hidetsune TAmaoki, PhD., and \\ Sadamasa Minato, Ph.D.
}

\section{SUMmARY}

Acute ligation of the left coronary artery of rats produced abnormal Q-wave in the electrocardiogram, tachycardia and frequent ventricular fibrillation. Serum CPK level was elevated, reaching a maximum at 3 to 5 hours after ligation and returning to near the pre-ligation level 24 hours later, when CPK activity in the left ventricle markedly decreased. Pretreatment with bucumolol at $2.5 \mathrm{mg} / \mathrm{Kg}$ s.c. and $5 \mathrm{mg} / \mathrm{Kg}$ s.c. lessened these changes and increased the survival rate in a dose related manner. dBucumolol at $5 \mathrm{mg} / \mathrm{Kg}$, on the other hand, increased survival rate primarily by suppressing ventricular fibrillation without any significant effect on other parameters. These results suggest that the membrane stabilizing action does not contribute to protective actions of bucumolol against myocardial ischemia.

\section{Additional Indexing Words:}

Myocardial CPK Abnormal $Q$ wave Ventricular fibrillation Membrane stabilizing action

Q ENEFICAL actions of $\beta$-adrenergic blocking agents on ischemic injury 1 produced by temporal occlusion of the coronary artery have been well established, ${ }^{1)-5}$ ) and the use of $\beta$-adrenergic blocking agents for the treatment of myocardial infarction scems pharmacologically reasonable. ${ }^{6}$ However, controversies exist as to whether the membrane stabilizing action of $\beta$-adrenergic blocking agents produces a potential hazard such as cardiac power failure. ${ }^{6)}$ In addition, conflicting results have been reported regarding d-isomer's preventive action against myocardial ischemic injury. ${ }^{51,7)}$

The present study was designed to determine if bucumolol, a potent $\beta$ adrenergic blocking agent with less membrane stabilizing activity than propranolol,8) would prevent myocardial injury produced by permanent occlu-

From the Cardiovascular Division, Central Research Laboratories and Fermentation Research Laboratories, Sankyo Co., Ltd., 1-2-58 Hiromachi, Shinagawa-ku, Tokyo 140, Japan.

Received for publication September 18, 1980.

Manuscript revised November 17, 1980. 
sion of the left coronary artery in rats and to asscss the role of the membrane stabilizing action of this agent. Since ventricular fibrillation and cardiac power failure are major causes of death in acute myocardial infarction, we evaluated the agent in terms of its ability to improve electrocardiographic as well as biochemical changes associated with myocardial ischemia.

\section{Methods}

Male Sprague-Dawley rats (Charles River) weighing 230 to $280 \mathrm{Gm}$ were intubated under ether anesthesia and ventilated artificially with air containing supplemental ether. A left thoracotomy was performed at the fifth intercostal space and the left coronary artery was ligated according to the method described by Selye et al. ${ }^{9)}$ Severe ventricular arrhythmia developed in all rats shortly after ligation of the left coronary artery. The chest was closed after this unstable period was over. For recording the electrocardiogram (Apex-Base lead), a pair of Ag-AgCl electrodes were sutured subcutaneously before the surgery; one at the right shoulder, the other at the left side of the chest above the sixth costchondral junction.

A total of 39 rats were divided into 5 groups. Five rats underwent a sham operation which involved the same procedures performed on the other 4 groups except that the left coronary artery was not ligated and no drug was given. Seventeen rats received saline injections to serve as controls. In the other 3 groups consisting of 6,5 , and 6 rats, dl-bucumolol $2.5 \mathrm{mg} / \mathrm{Kg}, 5 \mathrm{mg} / \mathrm{Kg}$ or d-bucumolol 5 $\mathrm{mg} / \mathrm{Kg}$ s.c. respectively were given $5 \mathrm{~min}$ prior to coronary ligation. Supplemental doses equal to half the initial doses were given every 3 hours for 9 hours.

Electrocardiogram was recorded prior to and 1, 3, 5, 7, 9, and 24 hours after coronary ligation. After each recording, a blood sample in a volume of $0.3 \mathrm{ml}$ was collected from the tail to determine serum creatine phosphokinase (CPK) activity. Twenty-four hours after coronary ligation the animals were killed and the heart was excised for myocardial CPK assay.

The free walls of the left and right ventricles and interventricular septum were separated and washed in cold saline. Tissue samples were homogenized in 0.25 M sucrose containing EDTA and mercaptoethanol, and centrifuged at $16,000 \mathrm{~g}$ for 10 min according to the method described by Kjekshus and Sobel. ${ }^{10)}$ The supernatant fraction was assayed for CPK activity with a commercial kit (SankyoCalbiochem). The activity was expressed as international units per $\mathrm{mg}$ of protein. Serum CPK was assayed in a volume of $0.002 \mathrm{ml}$ by the rate assay and expressed as international units per ml of serum.

\section{REsults}

\section{Ventricular fibrillation and mortality}

Immediately after the ligation of the coronary artery severe ventricular arrhythmias developed in all rats including those treated with bucumolol. However, most animals survived ventricular fibrillation with the aid of direct cardiac massage. After arrhythmias subsided the chest was closed and the 
electrocardiogram was periodically recorded. The time course for the changes in the electrocardiogram is shown in Fig. 1. After few hours of quiescent period ventricular fibrillation developed, the onset of which shown in Table I. The ventricular fibrillation often disappeared spontaneously or could be abolished by external resuscitation, i.e. by applying intermittent pressure to the chest. The occurrence of ventricular fibrillation was judged by behavioral changes, since in the preliminary study continuous recordings of the electro-

\section{Control}

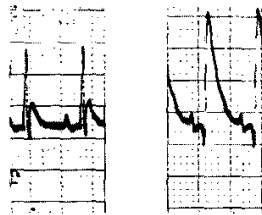

\section{Bucumolol}
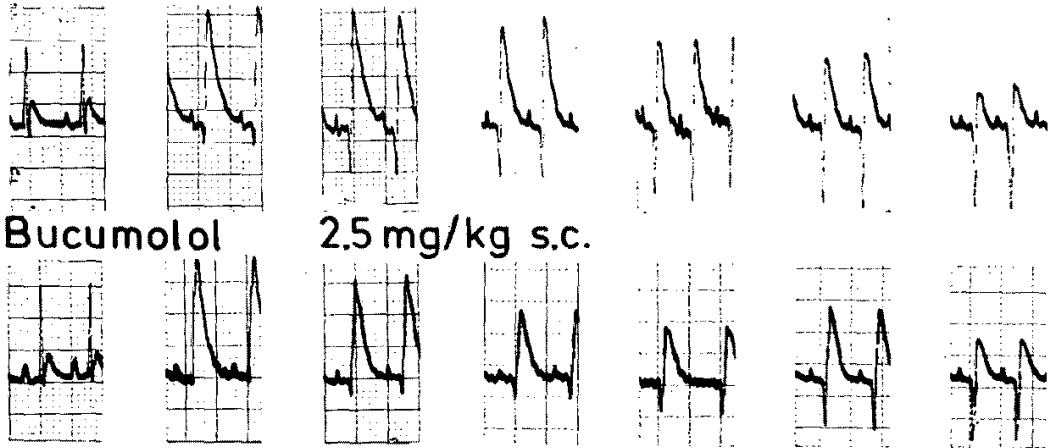

$2.5 \mathrm{mg} / \mathrm{kg}$ s.c.
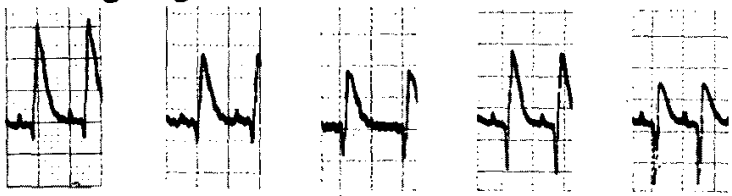

\section{d-Bucumolol}

\section{$5.0 \mathrm{mg} / \mathrm{kg}$ s.c.}

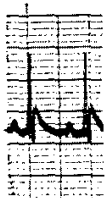

0

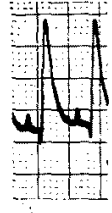

1

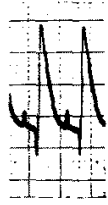

3

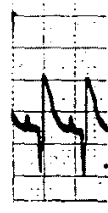

5

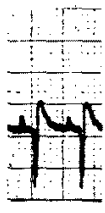

7

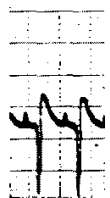

9

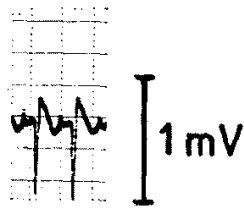

24

\section{hours after coronary ligation}

Fig. 1. Alteration of electrocardiogram (Lead I of apex-base leads) produced by coronary artery ligation in conscious rats.

Table I. Ventricular Fibrillation after Coronary Ligation as Affected by dl- and d-Bucumolol

\begin{tabular}{l|c|c|c|c}
\hline Drug & $\begin{array}{c}\text { Dose } \\
\mathrm{mg} / \mathrm{Kg} \\
\text { S.c. }\end{array}$ & $\begin{array}{c}\text { Incidence of } \\
\text { ventricular } \\
\text { fibrillation }\end{array}$ & $\begin{array}{c}\text { Onset of late ventricular } \\
\text { fibrillation (hrs) }\end{array}$ & Mortality \\
\hline Control & 2.5 & $16 / 17$ & $4.8 \pm 1.4 \mathrm{hrs}$ & $11 / 17$ \\
Bucumolol & 5.0 & $1 / 6$ & $10.7 \pm 4.3^{*}$ & $0 / 6$ \\
Bucumolol & 5.0 & $3 / 6$ & $19.2 \pm 3.3^{*}$ & $0 / 5$ \\
d-Bucumolol & $1 / 5$ & $14.4 \pm 4.3^{*}$ & $1 / 6$
\end{tabular}

* significant at $\mathrm{p}<0.05$ by non-paired t-test 
cardiogram revealed that the development of ventricular fibrillation was always accompanied by a seizure-like behavior including jumping and intense rigidity throughout a whole body. In the control group one rat did not develop ventricular fibrillation although it had an abnormal Q-wave similar to that seen in other control rats.

Pretreatment with dl-bucumolol at 2.5 and $5.0 \mathrm{mg} / \mathrm{kg}$ s.c. delayed the onset of late ventricnlar fibrillation and decreased the incidence of ventricular fibrillation in a dose related manner (Table I). d-Isomer of bucumolol at $5.0 \mathrm{mg} / \mathrm{Kg}$ s.c. was less potent than racemic mixture at the same dose. Eleven out of 17 control rats died of ventricular fibrillation within 24 hours while most rats in the dl- and d-bucumolol groups survived.

\section{Heart rate and electrocardiogram}

In the control group heart rate increased after coronary ligation (Fig. 2). dl-Bucumolol not only prevented the increase in heart rate, it lowered heart rate far below pre-ligation levels. In the d-bucumolol group heart rate decreased 1 and 7 hours after ligation; in other words, only when the animals were given the agent 1 hour before the electrocardiogram was recorded.

As shown in Fig. 1, changes in the electrocardiogram included an increase in R wave height followed by a decrease, an elevation of ST segment and development of an abnormal Q-wave that was not seen in normal rats. However, the ST segment in the ischemic rat heart was hardly distinguishable from the QRS complex. The Q-wave, the depth of which could easily be measured, appeared as early as 1 hour after coronary ligation in all control

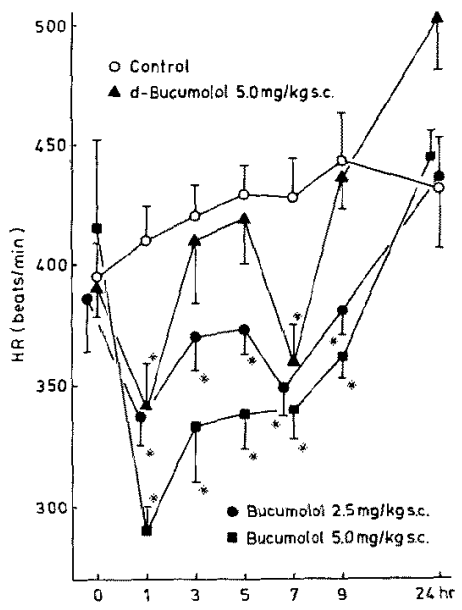

Fig. 2. Changes in heart rate following ligation of left coronary artery in conscious rats. Asterisks indicate a significant difference $(p<0.05)$ between the control and the other groups. 


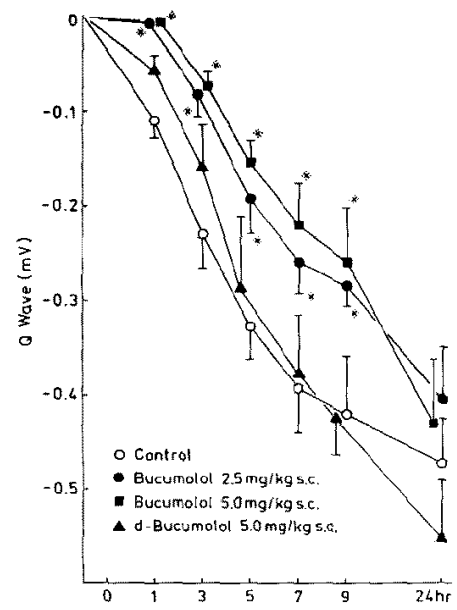

Fig. 3. Time course for the development of $Q$-wave following coronary artery ligation. Asterisks indicate a significant difference $(p<0.05)$ between the control and the other groups.

rats and grew with time, resulting in the so-called QR pattern. dl-Bucumolol both at lower and higher doses almost completely suppressed the development of the Q-wave at 1 hour (Figs. 1 and 3). In these groups, Q-waves appearing after 3 hours were smaller in size than those present at 1 hour in the control group. For up to 9 hours the depth of $Q$-wave in the 2 groups was significantly smaller than that of the control group. However, this difference was not significant at 24 hours. d-Bucumolol on the other hand, failed to suppress the development of the $Q$-wave although it tended to reduce the depth of the Q-wave at 1 hour.

\section{Serum and myocardial $C P K$}

Serum CPK level increased following coronary ligation, reaching a maximum at 3 to 5 hours and returning to near the pre-ligation level 24 hours later (Fig. 4). Pretreatment with dl-bucumolol suppressed the increase of serum CPK whereas $d$-isomer did not. In the sham operation group, serum CPK activity increased to a lesser degree than in the other groups, suggesting that in the groups with coronary ligation most of the elevated serum CPK was of myocardial origin.

Ligation of the left coronary artery produced a marked reduction of CPK in the free wall of the left ventricle and a slight reduction in the intraventricular septum, but no reduction in the right ventricle (Table II). dlBucumolol at 2.5 and $5.0 \mathrm{mg} / \mathrm{Kg}$ s.c. prevented the loss of CPK in the left ventricle in a dose-related manner whereas d-isomer failed to do so. 


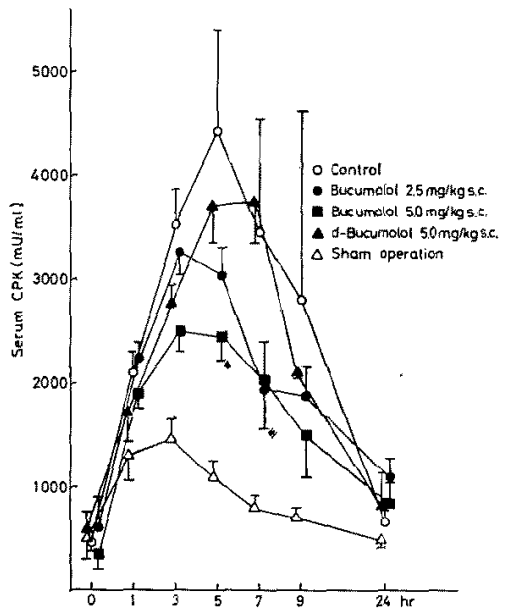

Fig. 4. Changes in serum CPK following coronary artery ligation. Asterisks indicate a significant difference $(p<0.05)$ between the control and the other groups with coronary artery occluded.

Table II. Effects of Bucumolol and Its d-Isomer on the Depletion of Myocardial CPK Produced by Ligation of Coronary Artery

\begin{tabular}{l|c|c|c|c}
\hline & \multicolumn{4}{|c}{ CPK activity (I.U./mg protein) } \\
\cline { 2 - 5 } & Left ventricle & Septum & Right ventricle & $\mathrm{N}$ \\
\hline Sham operation & $17.8 \pm 2.1^{*}$ & $19.0 \pm 0.5$ & $13.3 \pm 1.0$ & 5 \\
Control & $7.2 \pm 0.8$ & $14.5 \pm 2.9$ & $14.2 \pm 2.0$ & 6 \\
Bucumolol $2.5 \mathrm{mg} / \mathrm{Kg}$ & $10.2 \pm 3.1^{*}$ & $18.0 \pm 1.2$ & $13.6 \pm 0.8$ & 6 \\
Bucumolol & $12.2 \pm 0.7^{*}$ & $20.8 \pm 3.7$ & $14.6 \pm 1.1$ & 5 \\
d-Bucumolol $5.0 \mathrm{mg} / \mathrm{Kg}$ & $6.4 \pm 1.5$ & $15.3 \pm 2.3$ & $11.7 \pm 1.3$ & 5
\end{tabular}

* $\mathrm{p}<0.05$ vs control by non-paired $\mathrm{t}$-test

\section{Discussion}

Bucumolol is twice as potent as propranolol in $\beta$-adrenergic blocking action and one third as potent in membrane stabilizing action. ${ }^{8)}$ At doses of 2.5 and $5.0 \mathrm{mg} / \mathrm{Kg}$ s.c., dl-bucumolol completely blocks tachycardia produced by isoproterenol $0.3 \mu \mathrm{g} / \mathrm{Kg}$ i.v., whereas d-isomer at $5 \mathrm{mg} / \mathrm{Kg}$ s.c. produced virtually no $\beta$-blockade. dl-Bucumolol is equally as potent as its d-isomer in the membrane stabilizing action. ${ }^{11)}$ The membrane stabilizing action of $\mathrm{dl}$ bucumolol at $5 \mathrm{mg} / \mathrm{Kg}$ s.c. is manifested by the fact that d-bucumolol at the same dose prevented ventricular fibrillation (Table I).

Ventricular fibrillation developed immediately after coronary ligation was followed by a quiescent period of a few hours. This bimodal occurrence of 
ventricular fibrillation observed in the rat resembles the bimodal distribution of ventricular arrhythmias seen in the canine model by Harris. ${ }^{12)}$ However, the rat model differs from the canine model in that ventricular fibrillation in the rat often disappeared spontaneously or could be easily abolished by external resuscitation.

dl-Bucumolol suppressed the release of CPK from the ischemic left ventricle, delayed the development of abnormal Q-waves and decreased the incidence of ventricular fibrillations and the mortality in a dose-related manner. These actions are shared by other $\beta$-blockers including propranolol. But none of previous studies has evaluated the agents on 2 important criteria in the same animal model, i.e., ability to prevent ventricular fibrillations and to protect the myocardium from ischemic injury.

The present study does not provide enough information to speculate on the underlying mechanism causing the beneficial actions of dl-bucumolol. But sustained bradycardia produced by dl-bucumolol, but not by d-bucumolol, may account for the dl-bucumolol's preventive action against myocardial ischemia. The importance of bradycardia has been suggested by a number of investigators. ${ }^{4), 13), 14)}$ In addition, Lefer et $\mathrm{al}^{31}$ have demonstrated that timolol decreased the ischemia-induced elevation of the ST segment in dogs without significant effect on cardiac contractility. The failure of dbucumolol to reduce injury may be interpreted on the basis of the short duration of its cardiac slowing action (Fig. 2).

Regarding the effectiveness of d-propranolol in salvaging ischemic myocardium, conflicting results have been reported. Berdeaux et al ${ }^{5)}$ have demonstrated that d-propranolol reduced the ST segment elevation produced by coronary occlusion and suggested the involvement of the membrane stabilizing as well as negative inotropic actions in the beneficial actions of propranolol. On the other hand, Reimer et al ${ }^{7}$ presented the negative data. In the present study d-bucumolol, at a dose sufficient to prevent ventricular fibrillations, failed to reduce the ischemic injury. This suggests that the prevention of ventricular fibrillation per se does not contribute to the protection of the myocardium from ischemic injury. On the other hand, dl-bucumolol at $5.0 \mathrm{mg} / \mathrm{Kg}$ was more effective than $\mathrm{d}$-isomer at the same dose in preventing ventricular fibrillations (Table I), suggesting that $\beta$-blockade per se, in addition to the membrane stabilizing action, contributes to the antiarrhythmic action of the racemic mixture. ${ }^{15)}$

Of note is a finding that there was no difference between the control and bucumolol treated groups in the Q-wave size 24 hours after the ligation despite a significant difference in myocardial CPK loss (Figs. 1, 3 and Table II). Although the reason for this discrepancy remains to be solved this finding sug- 
gests that the Q-wave depth in an early period after coronary ligation is a more reliable index of myocardial injury than that in a later period, at least in this model.

In conclusion, dl-bucumolol protects the myocardium from ischemic injury mainly by its $\beta$-adrenergic blocking action. The membrane stabilizing action of the agent does not contribute to the protection against ischemia. On the other hand, prevention by dl-bucumolol of ventricular fibrillation is largely due to its membrane stabilizing action though $\beta$-adrenergic blocking action may partly be involved.

\section{ACKNOWLEDGMENT}

The authors are grateful for the pertinent advice of Dr. Koroku Hashimoto throughout this study.

\section{REFERENCES}

1. Reimer KA, Rasmussen MM, Jennings RB: Reduction by propranolol of myocardial necrosis following temporary coronary artery occlusion in dogs. Circulat Res 33: 353, 1973

2. Hills LD, Askenazi J, Braunwald E, Radvany P, Muller JE, Fishbein MC, Maroko PR: Use of changes in the epicardial QRS complex to assess interventions which modify the extent of myocardial necrosis following coronary artery occlusion. Circulation 53: 591, 1976

3. Lefer AM, Cohn JR, Osman GH: Protective action of timolol in acute myocardial ischemia. Eur J Pharmac 41: 379, 1977

4. Berdeaux A, Boissier JR, Giudicelli JE: Effect of atenolol on regional myocardial blood flow and ST segment elevation in the canine myocardium. Brit J Pharmac 60: 433, 1977

5. Berdeaux A, Da Costa CP, Garnier M, Boissier JR, Giudicelli JF: Beta adrenergic blockade, regional left ventricular blood flow and ST-segment elevation in canine experimental myocardial ischemia. J Pharmacol Exper Ther 205: 646, 1978

6. Lee RJ: Beta-adrenergic blockade in acute myocardial infarction. Life Sci 23: 2539, 1978

7. Reimer KA, Rasmussen MM, Jennings, RB: On the nature of protection by propranolol against myocardial necrosis after temporary coronary occlusion in dogs. Am J Cardiol 37: 520,1976

8. Oshima $T$, Kumakura $S$, Koike $H$, Nakayama K: Pharmacology of d1-5-methyl-8-(2hydroxy-3-t-butylaminopropoxy) coumarin hydrochloride (CS-359), a new $\beta$-adrenergic blocking agent. Jpn J Pharmac 23: 497, 1973

9. Selye H, Bajusz E, Grasso S, Mendell P: Simple techniques for the surgical acclusion of coronary vessels in the rat. Angiology $11: 398,1960$

10. Kjekshus JK, Sobel BE: Depressed myocardial creatinine phosphokinase activity following experimental myocardial infarction in rabbit. Circulat Res 27: 403, 1970

11. Nakayama $\mathrm{K}$, Oshima $\mathrm{T}$, Koike $\mathbf{H}$ : Antiarrhythmic activity of 5-methyl-8-(2-hydroxy-3t-butylaminopropoxy) coumarin hydrochloride (bucumolol), a $\beta$-adrenergic blocking agent, on aconitine-induced atrial and ouabain-induced ventricular arrhythmias in dogs. Jpn. J Pharmacol 29: 935, 1979

12. Harris AS: Delayed development of ventricular ectopic rhythms following experimental coronary occlusion. Circulation 1: 1318, 1950.

13. Redwood DR, Smith ER, Epstein SE: Coronary occlusion in the conscious dog. Effects of 
alteration in heart rate and arterial pressure on the myocardial ischcmia. Circulation 46 : 323,1972

14. Brown BG, Gundel WD, Gott VL, Covell JW: Coronary collateral flow following acute coronary occlusion: a diastolic phenomenon. Cardiovas. Res 8: 621, 1974

15. Corr PB, Gillis RA: Autonomic neural influence on dysrhythmias resulting from myocardial infarction. Circulat Res 43:1, 1978 